\title{
ON THE OSCILLATION OF SUMS OF RANDOM VARIABLES $\left(^{1}\right)$
}

BY

\section{ROSENBLATT}

1. Introduction. Let $X$ be a random variable with distribution function $F(x)$ and characteristic function $\phi(z)=\int_{-\infty}^{\infty} e^{i z x} d F(x)$. The sequence of partial sums $\left\{S_{n}\right\}$ will be said to be generated by $X$ if $S_{n}=\sum_{j=1}^{n} X_{j}$ where $X_{1}, \cdots$, $X_{n}, \cdots$ are independent, identically distributed random variables with distribution function $F(x)$.

Let the abbreviations i.o. and f.o. denote the phrases "infinitely often" and "finitely often," respectively. The sequence $\left\{S_{n}\right\}$ generated by the random variable $X$ is said to oscillate if

$$
P\left\{S_{n}>0 \text { i.o. }\right\}=P\left\{S_{n} \leqq 0 \text { i.o. }\right\}=1 .
$$

A sufficient condition for oscillation of the sequence $S_{n}$, convenient for the application of results concerning partial limit laws of normed sums of independent and identically distributed random variables, will be obtained. When $E(|X|)<\infty$, the problem considered will be shown to be equivalent to a problem dealt with by K. L. Chung and W. H. J. Fuchs [1](2).

The necessary and sufficient condition for the oscillation of the sequence $\left\{S_{n}\right\}$ generated by $X$ will be obtained. The necessary and sufficient condition is used to obtain a class of random variables each of which generates sums $S_{n}$ which satisfy

$$
\begin{aligned}
P\left\{\lim _{n \rightarrow \infty}\left|S_{n}\right|=\infty\right\} & =P\left\{\liminf _{n \rightarrow \infty} S_{n}=-\infty\right\} \\
& =P\left\{\limsup _{n \rightarrow \infty} S_{n}=\infty\right\}=1
\end{aligned}
$$

and

$$
\lim _{n \rightarrow \infty} P\left\{S_{n}>0\right\}=0
$$

simultaneously.

\section{Preliminary results.}

LEMMA 2.1. If $\lim \sup _{n \rightarrow \infty} P\left\{S_{n}>0\right\}>0\left(\lim \sup _{n \rightarrow \infty} P\left\{S_{n}<0\right\}>0\right)$, then $P\left\{S_{n}>0\right.$ i.o. $\}=1\left(P\left\{S_{n}<0\right.\right.$ i.o. $\left.\}=1\right)$.

Presented to the Society, April 27, 1951; received by the editors June 1, 1951.

(1) This work has been carried out under ONR contract.

(2) Numbers in brackets refer to the references at the end of the paper 
Now $\left\{S_{n}<0\right.$ i.o. $\}=\prod_{m=1}^{\infty} \bigcup_{n=m}^{\infty} \quad\left\{S_{n}>0\right\}$. But $P\left\{\bigcup_{n=m}^{\infty}\left\{S_{n}>0\right\}\right\}$ $\geqq \lim \sup _{n \rightarrow \infty} P\left\{S_{n}>0\right\}>0$. Since $\bigcup_{n=m}^{\infty}\left\{S_{n}>0\right\}$ is a monotone decreasing sequence of events,

$$
P\left\{S_{n}>0 \text { i.o. }\right\}=\lim _{m \rightarrow \infty} P\left\{\bigcup_{n=m}^{\infty}\left\{S_{n}>0\right\}\right\}>0 .
$$

P. Lévy has shown (see $\left[4\right.$, p. 147]) that if $A_{1}, A_{2}, \cdots$ are given constants,

$$
P\left\{S_{n}>A_{n} \text { i.o. }\right\}
$$

can be only either zero or one. Hence

$$
P\left\{S_{n}>0 \text { i.o. }\right\}=1 \text {. }
$$

One shows that $\lim \sup _{n \rightarrow \infty} P\left\{S_{n}<0\right\}>0$ implies $P\left\{S_{n}<0\right.$ i.o. $\}=1$ in like manner.

K. L. Chung and W. H. J. Fuchs [1] have studied recurrent values of partial sums $S_{n}$ generated by a random variable $X$. The value $b$ is said to be recurrent if

$$
P\left\{\left|S_{n}-b\right|<\epsilon \text { i.o. }\right\}=1
$$

for every $\epsilon>0$. They have shown that the set of recurrent values is a closed additive group. In particular, 0 is the one and only recurrent value if and only if $X=0$ with probability 1 .

LEMMA 2.2. If the partial sums $S_{n}$ generated by $X$ have recurrent values and if $X \neq 0$ with positive probability, the sequence $\left\{S_{n}\right\}$ oscillates.

Zero cannot be the only recurrent value since $X \neq 0$ with positive probability. There must then be two values $a>0, b<0$ which are recurrent values, that is, given any $\epsilon>0$

$$
P\left\{\left|S_{n}-b\right|<\epsilon \text { i.o. }\right\}=P\left\{\left|S_{n}-a\right|<\epsilon \text { i.o. }\right\}=1 .
$$

Let $\epsilon<\min (a,|b|)$. Then

$$
\begin{aligned}
& P\left\{S_{n}>0 \text { i.o. }\right\}=P\left\{\left|S_{n}-a\right|<\epsilon \text { i.o. }\right\}=1, \\
& P\left\{S_{n}<0 \text { i.o. }\right\}=P\left\{\left|S_{n}-b\right|<\epsilon \text { i.o. }\right\}=1 .
\end{aligned}
$$

Theorem 2.1. If $E(|X|)<\infty$ and $X \neq 0$ with positive probability, the problem of oscillation and the Chung-Fuchs problem are equivalent. Under these conditions, oscillation takes place if and only if $E(X)=0$.

If $X \neq 0$ with positive probability, Lemma 2.2 implies that the ChungFuchs problem is included in the problem of oscillation. Chung and Fuchs [1] have shown that if $E(|X|)<\infty$ and $E(X)=0$, there are recurrent values and hence there is oscillation. But if $E(X)=m \neq 0$, the strong law of large numbers tells us that 


$$
P\left\{\lim _{n \rightarrow \infty} \frac{S_{n}}{n}=m\right\}=1
$$

and hence (1.1) cannot be true. The equivalence of the two problems when $E(|X|)<\infty$ is demonstrated.

If $f(z)$ is a complex-valued function, let $\operatorname{Re} f(z)$ and $\operatorname{Im} f(z)$ denote the real and imaginary parts of $f(z)$, respectively.

Let

$$
\log _{2} z=\log \{-\log z\} \text { and } \log _{n} z=\log \left\{\log _{n-1} z\right\}, \quad n>2 .
$$

LEMMA 2.3. The sequence $\left\{S_{n}\right\}$ generated by. $X$ has no recurrent values if the characteristic function $\phi(z)$ of $X$ is such that

$$
-\liminf _{z \rightarrow 0+} \frac{1-\operatorname{Re} \phi(z)}{z \log z \cdots \log _{n-1} z \log _{n}^{1+\epsilon} z}>k>0
$$

for some $\epsilon>0$ and an integer $n \geqq 1$.

A necessary and sufficient condition that there be recurrent values [1] is that

$$
\lim _{\rho \rightarrow 1-} \int_{-\delta}^{\delta} \frac{1}{1-\rho \phi(z)} d z=\infty, \quad \delta>0 .
$$

But

$$
\begin{aligned}
\lim _{\rho \rightarrow 1-} \int_{-\delta}^{\delta} \frac{1}{1-\rho \phi(z)} d z & =\lim _{\rho \rightarrow 1-} \int_{-\delta}^{\delta} \frac{1-\rho \operatorname{Re} \phi(z)}{(1-\rho \operatorname{Re} \phi(z))^{2}+(\rho \operatorname{Im} \phi(z))^{2}} d z \\
& \leqq \lim _{\rho \rightarrow 1-} \int_{-\delta}^{\delta} \frac{1}{1-\rho \operatorname{Re} \phi(z)} d z \\
& \leqq \int_{-\delta}^{\delta} \frac{1}{1-\operatorname{Re} \phi(z)} d z .
\end{aligned}
$$

But this last integral converges if $(2.1)$ is satisfied.

Lemma 2.4. A necessary and sufficient condition that $S_{n}$ oscillate is that

$$
\sum_{j=1}^{\infty} P\left\{S_{j}>0, S_{j+1} \leqq 0\right\}=\infty
$$

and

$$
\sum_{j=1}^{\infty} P\left\{S_{i} \leqq 0, S_{j+1}>0\right\}=\infty .
$$

The necessity is trivial since the theorem of Borel-Cantelli tells us that 


$$
\sum_{j=1}^{\infty} P\left\{S_{i}>0, S_{j+1} \leqq 0\right\}<\infty
$$

or

$$
\sum_{j=1}^{\infty} P\left\{S_{i} \leqq 0, S_{i+1}>0\right\}<\infty
$$

implies that there is no oscillation with probability 1 .

Now

$$
\begin{aligned}
P\left\{S_{n}>0 \text { f.o. }\right\}= & P\left\{S_{n} \leqq 0 ; n=1,2, \cdots\right\} \\
& +\sum_{j=1}^{\infty} P\left\{S_{i}>0, S_{j+n} \leqq 0 ; n=1,2, \cdots\right\} \\
\geqq & P\left\{S_{n} \leqq 0 ; n=1,2, \cdots\right\} \\
& +\sum_{j=1}^{\infty} P\left\{S_{j}>0, S_{j+1} \leqq 0, S_{j+n}-S_{j+1} \leqq 0 ; n=1,2, \cdots\right\} \\
= & P\left\{S_{n} \leqq 0 ; n=1,2, \cdots\right\} \\
& +\sum_{j=1}^{\infty} P\left\{S_{j}>0, S_{j+1} \leqq 0\right\} P\left\{S_{n} \leqq 0 ; n=1,2, \cdots\right\} \\
= & P\left\{S_{n} \leqq 0 ; n=1,2, \cdots\right\}\left[1+\sum_{j=1}^{\infty} P\left\{S_{j}>0, S_{j+1} \leqq 0\right\}\right] .
\end{aligned}
$$

The divergence of $\sum_{j=1}^{\infty} P\left\{S_{j}>0, S_{j+1} \leqq 0\right\}$ implies that $P\left\{S_{n} \leqq 0\right.$; $n=1,2, \cdots\}=0$. But

$$
P\left\{S_{n}>0 \text { f.o. }\right\} \leqq \lim _{m \rightarrow \infty} P\left\{S_{n} \leqq 0 ; n=1,2, \cdots\right\} \cdot\left[1+\sum_{j=1}^{m} P\left\{S_{j}>0\right\}\right] .
$$

Hence the divergence of $\sum_{j=1}^{\infty} P\left\{S_{j}>0, S_{j+1} \leqq 0\right\}$ implies that

$$
P\left\{S_{n}>0 \text { f.o. }\right\}=0 \text {. }
$$

An analogous argument indicates that

$$
\sum_{j=1}^{\infty} P\left\{S_{j} \leqq 0, S_{j+1}>0\right\}=\infty \text { implies that } P\left\{S_{n} \leqq 0 \text { f.o. }\right\}=0 .
$$

3. Application of the sufficient condition. One makes use of Lemma 2.1 and the study of the infinitely divisible laws as partial limit laws of normed sums of independent random variables to characterize a class of random variables each of which generates partial sums $S_{n}$ that oscillate. Random variables with no finite first moment are of interest since Chung and Fuchs have completely solved the problem for random variables having a first moment. 
THEOREM 3.1. Let the sequence $S_{n}$ be generated by a random variable $X$ with distribution function $F(x)$. If there are monotone sequences $n_{k} \rightarrow \infty$ and $a_{k} \rightarrow \infty$ of positive integers and positive numbers respectively such that

(i) $\quad \lim _{\epsilon \rightarrow 0} \lim _{k \rightarrow \infty} \frac{n_{k}}{a_{k}}\left[\int_{|x|<\epsilon a_{k}} x d F(x)+\int_{|x|<\epsilon a_{k}} \frac{x}{1+\left(x / a_{k}\right)^{2}} d F(x)\right]=m$,

(ii) $\lim _{\epsilon \rightarrow 0} \lim _{k \rightarrow \infty} \frac{n_{k}}{a_{k}^{2}}\left[\int_{|x|<\epsilon a_{k}} x^{2} d F(x)-\left(\int_{|x|<\epsilon a_{k}} x d F(x)\right)^{2}\right]=\sigma^{2}$,

(iii) $\lim _{k \rightarrow \infty} n_{k}\left(1-F\left(x a_{k}\right)\right)=-\Omega(x)$, $x>0$,

$\lim _{x \rightarrow \infty} n_{k} F\left(-x a_{k}\right)=\Omega(-x)$, $x>0$,

where $\Omega(x)$ is finite for $|x|>0$ and $\Omega(-\infty)=\Omega(\infty)=0$,

(iv) . $\Omega(x)$ increases somewhere in both the ranges $x>0$ and $x<0$,

then the sequence $\left\{S_{n}\right\}$ oscillates.

W. Doeblin [2] has shown that if conditions (i), (ii), (iii) are satisfied, the normed subsequence $S_{n_{k}} / a_{k}$ has a limiting distribution function as $k \rightarrow \infty$ whose characteristic function is

$$
\exp \left\{i m z-\frac{\sigma^{2}}{2} z+\int_{-\infty}^{\infty}\left(e^{i z x}-1-\frac{i z x}{1+x^{2}}\right) d \Omega(x)\right\} .
$$

Note that $\Omega(x)$ is nondecreasing in the ranges $x>0$ and $x<0$. If $\Omega(x)$ increases somewhere in both the ranges $x>0$ and $x<0$, the limiting distribution function attributes positive probability to both positive and negative values, that is,

$$
\lim _{k \rightarrow \infty} P\left\{\frac{S_{n_{k}}}{a_{k}}>0\right\}>0, \quad \lim _{k \rightarrow \infty} P\left\{\frac{S_{n_{k}}}{a_{k}}<0\right\}>0 .
$$

This follows from the fact that the characteristic function

$$
\exp \left\{\int_{-\infty}^{\infty}\left(e^{i z x}-1-\frac{i z x}{1+x^{2}}\right) d \Omega(x)\right\}
$$

is the limit of a sequence each element of which is the characteristic function of a weighted sum of properly centered independent and non-identically distributed Poisson random variables (see [4, pp. 173-180]).

But then Lemma 2.1 implies that the sequence $\left\{S_{n}\right\}$ oscillates.

4. Application of the necessary and sufficient condition. The object of this section is to obtain Theorems 4.2 and 4.3 which give a characterization of a class of random variables satisfying (1.2) and (1.3) simultaneously. 
LеммA 4.1. Let $F(x)$ be a distribution function with $\int|x| \alpha d F(x)<\infty$ for some $\alpha, 0<\alpha<1$. Given $Y>0$, let

$$
F(x ; Y)=\left\{\begin{array}{lll}
0 & \text { if } & x \leqq-Y, \\
F(x)-F(-Y) & \text { if } & |x|<Y, \\
F(Y)-F(-Y) & \text { if } & x \geqq Y,
\end{array}\right.
$$

and $\phi(z ; Y)$ be the Fourier-Stieltjes transform of $F(x ; Y)$. Then $\operatorname{Im} \phi(z ; Y)$ $=o\left(|z|^{\alpha}\right)$ at $z=0$ uniformly for all $Y>0$.

Now $|\operatorname{Im} \phi(z, Y)|=\int_{|x|<Y} \sin z x d F(x) \leqq \int_{|x|<Y}|x z| \alpha|\sin x z|^{1-\alpha} d F(x)$ $=|z|^{\alpha} o(1)$ uniformly for all $Y>0$ by the Lebesgue theorem on dominated convergence.

Let $X$ be a lattice random variable, i.e., there is a largest $h>0$ such that

$$
P\{X=k h\}=p_{k} \geqq 0, \quad k=0, \pm 1, \pm 2, \cdots,
$$

and

$$
\sum_{k=-\infty}^{\infty} p_{k}=1
$$

Let

$$
\phi_{1}(z)=\sum_{j=-\infty}^{-1} p_{j} e^{i j h z}, \quad \phi_{2}(z)=\sum_{j=1}^{\infty} p_{j} e^{i j h z} .
$$

THEOREM 4.1. Let $X$ be a lattice random variable with $E\left(|X|^{\alpha}\right)<\infty$ for some $\alpha, 0<\alpha<1$, and let the partial sums generated by $X$ have no recurrent values. $A$ necessary and sufficient condition that the partial sums generated by $X$ oscillate is that

$$
\begin{gathered}
\lim _{\rho \rightarrow 1-}\left[\frac{h(1-\rho)}{4 \pi} \int_{-\pi / h}^{\pi / h} \frac{\sin z h}{1-\cos z h} \frac{\operatorname{Im} \phi_{2}(z)}{|1-\rho \phi(z)|^{2}} d z\right. \\
+\frac{\rho h}{4 \pi} \int_{-\pi / h}^{\pi / h} \frac{\sin z h}{1-\cos z h}\left(\frac{\left\{\phi_{1}(0)-\operatorname{Re} \phi_{1}(z)\right\} \operatorname{Im} \phi_{2}(z)}{|1-\rho \phi(z)|^{2}}\right. \\
\left.=\lim _{\rho \rightarrow 1-}\left[-\frac{\left\{\phi_{2}(0)-\operatorname{Re} \phi_{2}(z)\right\} \operatorname{Im} \phi_{1}(z)}{|1-\rho \phi(z)|^{2}}\right) d z\right] \\
+\frac{\rho(1-\rho)}{4 \pi} \int_{-\pi / h}^{\pi / h} \frac{\sin z h}{1-\cos z h} \frac{\operatorname{Im} \phi_{1}(z)}{1-\left.\rho \phi(z)\right|^{2}} d z \\
\frac{\sin z h}{1-\cos z h}\left(\frac{\left\{\phi_{1}(0)-\operatorname{Re} \phi_{1}(z)\right\} \operatorname{Im} \phi_{2}(z)}{|1-\rho \phi(z)|^{2}}\right. \\
\left.\left.-\frac{\left\{\phi_{2}(0)-\operatorname{Re} \phi_{2}(z) \operatorname{Im}_{\phi_{1}}(z)\right.}{|1-\rho \phi(z)|^{2}}\right) d z\right]=\infty .
\end{gathered}
$$


Lemma 2.4 implies that

$$
\begin{aligned}
& \lim _{\rho \rightarrow 1-} \sum_{j=1}^{\infty} \rho^{j} P\left\{S_{j}>0, S_{j+1} \leqq 0\right\}=\infty, \\
& \lim _{\rho \rightarrow 1-} \sum_{j=1}^{\infty} \rho^{j} P\left\{S_{j} \leqq 0, S_{j+1}>0\right\}=\infty
\end{aligned}
$$

is a necessary and sufficient condition for oscillation.

First consider (4.3).

$$
\sum_{j=1}^{\infty} \rho^{i} P\left\{S_{j}>0, S_{j+1} \leqq 0\right\}=\sum_{j=1}^{\infty} \rho^{j} \sum_{k=1}^{\infty} P\left\{S_{j}=k h\right\} \sum_{n=-\infty}^{-k} P\{X=n h\} .
$$

But $P\left\{S_{j}=k h\right\}=(h / 2 \pi) \int_{-\pi / h}^{\pi / h} \phi^{i}(z) e^{-i k h z} d z$ where $\phi(z)$ is the characteristic function of $X$. Expression (4.5) can then be rewritten as

$$
\begin{aligned}
\sum_{j=1}^{\infty} \rho^{j} \sum_{k=1}^{\infty} \frac{h}{2 \pi} \int_{-\pi / h}^{\pi / h} \phi^{i}(z) e^{-i k h z} d z \sum_{n=-\infty}^{-k} p_{n} \\
=\sum_{k=1}^{\infty} \frac{h}{2 \pi} \int_{-\pi / h}^{\pi / h} e^{-i k h z} \frac{\rho \phi(z)}{1-\rho \phi(z)} d z \sum_{j=-\infty}^{-k} p_{i} \\
=\sum_{j=-1}^{-\infty} \sum_{k=1}^{-j} \frac{h}{2 \pi} \int_{-\pi / h}^{\pi / h} e^{-i k h z} \frac{\rho \phi(z)}{1-\rho \phi(z)} d z p_{j} \\
=\sum_{j=-1}^{-\infty} \frac{h}{2 \pi} \int_{-\pi / h}^{\pi / h} \frac{e^{-i h z}-e^{i(j-1) h z}}{1-e^{-i h z}} p_{j} \frac{\rho \phi(z)}{1-\rho \phi(z)} d z \\
=\frac{h}{2 \pi} \int_{-\pi / h}^{\pi / h} \frac{e^{-i h z}\left\{\phi_{1}(0)-\phi_{1}(z)\right\}}{1-e^{-i h z}} \frac{\rho \phi(z)}{1-\rho \phi(z)} d z
\end{aligned}
$$

where the last interchange of summation and integration follows by applying Lemma 4.1. Now expression (4.6) can be rewritten as

$$
\begin{aligned}
& -\frac{h}{2 \pi} \int_{-\pi / h}^{\pi / h}\left\{\phi_{1}(0)-\phi_{1}(z)\right\} \frac{\rho \phi(z)}{1-\rho \phi(z)} d z \\
& +\frac{h}{2 \pi} \int_{-\pi / h}^{\pi / h} \frac{\phi_{1}(0)-\phi_{1}(z)}{1-e^{-i h z}} \frac{\rho \phi(z)}{1-\rho \phi(z)} d z .
\end{aligned}
$$

But expression (4.7) equals

$$
-P\{X<0\} \sum_{n=1}^{\infty} \rho^{n} P\left\{S_{n}=0\right\}+\sum_{j=-1}^{-\infty} p_{i} \sum_{n=1}^{\infty} \rho^{n} P\left\{S_{n}=-j h\right\}
$$

which is bounded by 


$$
\sum_{j=1}^{\infty} P\left\{S_{j}=0\right\}<\infty
$$

in absolute value. The assumption that the partial sums have no recurrent values implies that $\sum_{j=1}^{\infty} P\left\{S_{j}=0\right\}<\infty$ [1]. Hence we need consider only (4.8) which can be written as

$$
\begin{aligned}
& -\frac{h}{2 \pi} \int_{-\pi / h}^{\pi / h} \frac{\phi_{1}(0)-\phi_{1}(z)}{1-e^{-i h z}} d z \\
& +\frac{h}{2 \pi} \int_{-\pi / h}^{\pi / h} \frac{\phi_{1}(0)-\phi_{1}(z)}{1-e^{-i h z}} \frac{1}{1-\rho \phi(z)} d z .
\end{aligned}
$$

But (4.9) equals

$$
-P\{X<0\}
$$

so that only (4.10) need be considered. Expression (4.10) equals

$$
\begin{aligned}
& \frac{h}{4 \pi} \int_{-\pi / h}^{\pi / h} \frac{\phi_{1}(0)-\phi_{1}(z)}{1-\rho \phi(z)} d z \\
- & \frac{h i}{4 \pi} \int_{-\pi / h}^{\pi / h} \frac{\sin z h}{1-\cos z h} \frac{\phi_{1}(0)-\phi_{1}(z)}{1-\rho \phi(z)} d z .
\end{aligned}
$$

But (4.11) is bounded in absolute value by

$$
1+\sum_{j=1}^{\infty} P\left\{S_{j}=0\right\}<\infty
$$

so that only (4.12) need be considered. Expression (4.12) can be rewritten as

$$
\begin{aligned}
& -\frac{h i}{4 \pi} \int_{-\pi / h}^{\pi / h} \frac{\sin z h}{1-\cos z h} \frac{\phi_{1}(0)-\phi_{1}(z)}{|1-\rho \phi(z)|^{2}}(1-\rho \Phi(z)) d z \\
= & -\frac{h i}{4 \pi} \int_{-\pi / h}^{\pi / h} \frac{\sin z h}{1-\cos z h} \frac{\phi_{1}(0)-\phi_{1}(z)}{|1-\rho \phi(z)|^{2}}(1-\rho) d z \\
& -\frac{h i}{4 \pi} \int_{-\pi / h}^{\pi / h} \frac{\sin z h}{1-\cos z h} \frac{\phi_{1}(0)-\phi_{1}(z)}{|1-\rho \phi(z)|^{2}}(1-\Phi(z)) \rho d z \\
= & -\frac{h(1-\rho)}{4 \pi} \int_{-\pi / h}^{\pi / h} \frac{\sin z h}{1-\cos z h} \frac{\operatorname{Im} \phi_{1}(z)}{|1-\rho \phi(z)|^{2}} d z \\
& +\frac{\rho h}{4 \pi} \int_{-\pi / h}^{\pi / h} \frac{\sin z h}{1-\cos z h}\left(\frac{\left\{\phi_{1}(0)-\operatorname{Re} \phi_{1}(z)\right\} \operatorname{Im} \phi_{2}(z)}{|1-\rho \phi(z)|^{2}}\right. \\
& \left.-\frac{\left\{\phi_{2}(0)-\operatorname{Re} \phi_{2}(z)\right\} \operatorname{Im} \phi_{1}(z)}{|1-\rho \phi(z)|^{2}}\right) d z .
\end{aligned}
$$


Hence equation (4.3) is true if and only if the limit of (4.13) as $\rho \rightarrow 1-$ is infinite. One derives condition (4.1) in a completely analogous manner from equation (4.4).

Given the distribution function $F(x)$, consider the two auxiliary distribution functions

$$
F_{2}(x)=\left\{\begin{array}{lll}
0 & \text { if } & x \leqq 0, \\
\frac{F(x)}{1-F(0+)} & \text { if } & x>0
\end{array}\right.
$$

and

$$
F_{1}(x)= \begin{cases}\frac{F(x)}{F(0-)} & \text { if } \quad x<0, \\ 1 & \text { if } \quad x \geqq 0 .\end{cases}
$$

Let $\phi(z), \Phi_{1}(z), \Phi_{2}(z)$ be the characteristic functions of $F(x), F_{1}(x)$, and $F_{2}(z)$, respectively.

LEMMA 4.2. Let $F(x)$ be such that

$$
1-F_{2}(x)=x^{-\alpha} h_{2}(x), \quad F_{1}(x)=x^{-\beta} h_{1}(x),
$$

$0<\alpha, \beta<1$ where

$$
\lim _{x \rightarrow \infty} \frac{h_{2}(c x)}{h_{2}(x)}=\lim _{x \rightarrow-\infty} \frac{h_{1}(c x)}{h_{1}(x)}=1
$$

for every constant $c>0$. Then

$$
\frac{1-\operatorname{Re} \Phi_{2}(z)}{\Gamma(1-\alpha) \cos (\pi \alpha / 2)}, \quad \frac{\operatorname{Im} \Phi_{2}(z)}{\sin (\pi \alpha / 2) \Gamma(1-\alpha)} \sim 1-F_{2}\left(\frac{1}{z}\right) \quad \text { as } z \rightarrow 0+
$$

and

$$
\frac{1-\operatorname{Re} \Phi_{1}(z)}{\Gamma(1-\alpha) \cos (\pi \alpha / 2)}, \quad \frac{-\operatorname{Im} \Phi_{1}(z)}{\sin (\pi \alpha / 2) \Gamma(1-\alpha)} \sim F_{1}\left(-\frac{1}{z}\right) \quad \text { as } \quad z \rightarrow 0+.
$$

Let $\left\{a_{k}\right\},\left\{b_{k}\right\}$ be positive sequences such that

$$
1-F_{2}\left(a_{k}\right) \sim 1 / k, \quad F_{1}\left(-b_{k}\right) \sim 1 / k
$$

as $k \rightarrow \infty$. Let $Y_{1}, Y_{2}, \cdots$ be independent random variables with common distribution function $F_{2}(x)$, while $Z_{1}, Z_{2}, \cdots$, are independent random variables with common distribution function $F_{1}(x)$. Let $S_{n}$ and $T_{n}$ be their corresponding partial sums. Then $[2,3]$

$$
P\left\{S_{n} \leqq x a_{n}\right\} \rightarrow G_{\alpha}(x), \quad P\left\{T_{n} \leqq x b_{n}\right\} \rightarrow H_{\beta}(x)
$$


where $G_{\alpha}(x), H_{\beta}(x)$ have the characteristic functions $\gamma_{\alpha}(z), \delta_{\beta}(z)$ and

$$
\delta_{\beta}(z)=\bar{\gamma}_{\beta}(z), \gamma_{\alpha}(z)=\exp -\left\{|z|^{\alpha}\left(\cos \frac{\pi \alpha}{2}-i \sin \frac{\pi \alpha}{2} \operatorname{sgn} z\right) \Gamma(1-\alpha)\right\} .
$$

Then

$$
\begin{aligned}
n\left(1-\operatorname{Re} \Phi_{2}\left(z / a_{n}\right)\right) & \rightarrow z^{\alpha} \cos \frac{\pi \alpha}{2} \Gamma(1-\alpha), \\
n \operatorname{Im} \Phi_{2}\left(z / a_{n}\right) & \rightarrow z^{\alpha} \sin \frac{\pi \alpha}{2} \Gamma(1-\alpha)
\end{aligned}
$$

as $n \rightarrow \infty, z>0$. But the choice of $\left\{a_{k}\right\}$ and the properties of $h_{2}(x)$ imply that

$$
n\left(1-F_{2}\left(a_{n} x\right)\right) \rightarrow \frac{1}{x^{\alpha}}
$$

as $n \rightarrow \infty$. This implies that

$$
\frac{1-\operatorname{Re} \Phi_{2}(z)}{\cos (\pi \alpha / 2) \Gamma(1-\alpha)}, \quad \frac{\operatorname{Im} \Phi_{2}(z)}{\sin (\pi \alpha / 2) \Gamma(1-\alpha)} \sim 1-F_{2}\left(\frac{1}{z}\right)
$$

as $z \rightarrow 0+$. The analogous result for $1-\operatorname{Re} \Phi_{1}(z), \operatorname{Im} \Phi_{1}(z)$ follows in like manner.

TheOREM 4.2. Let $F(x)$ be such that

$$
1-F_{2}(x)=x^{-\alpha} h_{2}(x), \quad F_{1}(x)=x^{-\beta} h_{1}(x),
$$

$0<\alpha, \beta<1$, where

$$
\lim _{x \rightarrow \infty} \frac{h_{2}(c x)}{h_{2}(x)}=\lim _{x \rightarrow-\infty} \frac{h_{1}(c x)}{h_{1}(x)}=1
$$

for every constant $c>0$. There is no oscillation of the partial sums generated by a random variable $X$ with distribution function $F(x)$ if $\alpha \neq \beta$. Assume $\alpha=\beta$. Then a sufficient condition for the partial sums generated to oscillate is that there be $a$ positive integer $n$ and constants $k_{1}, k_{2}>0$ such that

$$
\frac{-k_{2}}{\log z \cdots \log _{n} z}<\frac{h_{1}(-1 / z)}{h_{2}(1 / z)}<-k_{1} \log z \cdots \log _{n} z
$$

as $z \rightarrow 0+$. On the other hand, if there is a positive integer $n$ and there are constants $\epsilon, k>0$ such that

$$
\frac{h_{1}(-1 / z)}{h_{2}(1 / z)}>-k \log z \cdots \log _{n-1} z \log _{n}^{1+\epsilon} z
$$


or

(C)

$$
\frac{h_{2}(1 / z)}{h_{1}(-1 / z)}>-k \log z \cdots \log _{n-1} z \log _{n}^{1+\epsilon} z
$$

as $z \rightarrow 0+$, there is no oscillation.

Let $X$ first be a lattice random variable. If $X$ has a distribution function of the form specified above, Lemmas 4.2 and 2.3 imply that there are no recurrent values. In investigating expressions (4.1) and (4.2) as $\rho \rightarrow 1-$, it is clear that one need only consider the indicated integrations over a neighborhood $(-\epsilon, \epsilon), \epsilon>0$, of zero. Lemma 4.2 implies that

$$
\frac{\phi_{2}(0)-\operatorname{Re} \phi_{2}(z)}{\cos (\pi \alpha / 2)}, \quad \frac{\operatorname{Im} \phi_{2}(z)}{\sin (\pi \alpha / 2)} \sim z^{\alpha} h_{2}\left(\frac{1}{z}\right) \Gamma(1-\alpha)(1-F(0+))
$$

and

$$
\frac{\phi_{1}(0)-\operatorname{Re} \phi_{1}(z)}{\cos (\pi \alpha / 2)}, \quad \frac{-\operatorname{Im} \phi_{1}(z)}{\sin (\pi \alpha / 2)} \sim z^{\beta} h_{1}\left(-\frac{1}{z}\right) \Gamma(1-\alpha) F(0-)
$$

as $z \rightarrow 0+$. Now

$$
\begin{aligned}
0 & \leqq-\frac{h(1-\rho)}{4 \pi} \int_{-\epsilon}^{\epsilon} \frac{\sin z h}{1-\cos z h} \frac{\operatorname{Im} \phi_{1}(z)}{|1-\rho \phi(z)|^{2}} d z \\
& \leqq-\frac{h}{4 \pi} \int_{-\epsilon}^{\epsilon} \frac{\sin z h}{1-\cos z h} \frac{\operatorname{Im} \phi_{1}(z)}{1-\operatorname{Re} \phi(z)} d z .
\end{aligned}
$$

The non-negativity of the integrand and Fatou's lemma imply that

$$
\begin{aligned}
& \liminf _{\rho \rightarrow 1-} \int_{-\epsilon}^{\epsilon} \frac{\sin z h}{1-\cos z h}\left(\frac{\left\{\phi_{1}(0)-\operatorname{Re} \phi_{1}(z)\right\} \operatorname{Im} \phi_{2}(z)}{|1-\rho \phi(z)|^{2}}\right. \\
&\left.-\frac{\left\{\phi_{2}(0)-\operatorname{Re} \phi_{2}(z)\right\} \operatorname{Im} \phi_{1}(z)}{|1-\rho \phi(z)|^{2}}\right) d z \\
& \geqq \int_{-\epsilon}^{\epsilon} \frac{\sin z h}{1-\cos z h}\left(\frac{\left\{\phi_{1}(0)-\operatorname{Re} \phi_{1}(z)\right\} \operatorname{Im} \phi_{2}(z)}{|1-\phi(z)|^{2}}-\frac{\left\{\phi_{2}(0)-\operatorname{Re} \phi_{2}(z)\right\} \operatorname{Im} \phi_{1}(z)}{|1-\phi(z)|^{2}}\right) d z .
\end{aligned}
$$

However

$$
\frac{1}{|1-\rho \phi(z)|^{2}} \leqq \frac{1}{(1-\operatorname{Re} \phi(z))^{2}},
$$$$
0<\rho \leqq 1
$$

But $|1-\phi(z)|$ and $1-\operatorname{Re} \phi(z)$ have the same behavior at zero as can be seen 
by making use of (4.14) and (4.15). Hence

$$
\int_{-\epsilon}^{\epsilon} \frac{\sin z h}{1-\cos z h} \frac{\left\{\phi_{1}(0)-\operatorname{Re} \phi_{1}(z)\right\} \operatorname{Im} \phi_{2}(z)-\left\{\phi_{2}(0)-\operatorname{Re} \phi_{2}\right\} \operatorname{Im} \phi_{1}(z)}{|1-\rho \phi(z)|^{2}} d z
$$

diverges as $\rho \rightarrow 1-$ if and only if

$$
\int_{0}^{\epsilon} \frac{1}{z} \frac{z^{\alpha+\beta} h_{2}(1 / z) h_{1}(-1 / z)}{z^{2 \alpha} h_{1}^{2}(-1 / z)+z^{2 \beta} h_{2}(1 / z)} d z
$$

diverges.

Let $\beta>\alpha$. By making use of (4.14) and (4.15), we see that (4.16) is bounded by a multiple of

$$
\int_{0}^{\epsilon} z^{\beta-\alpha-1} \frac{h_{1}(-1 / z)}{h_{2}(1 / z)} d z
$$

which converges since

$$
\frac{h_{1}(-1 / z)}{h_{2}(1 / z)}=o\left(z^{\gamma}\right)
$$

as $z \rightarrow 0+$ for every $\gamma<0$. Expression (4.17) converges for the same reason. Hence, (4.2) converges and there is no oscillation. If $\alpha>\beta$, a similar argument shows that (4.1) converges.

Let $\alpha=\beta$. Assume condition (C). Then both (4.16) and (4.17) are bounded by a multiple of

$$
-\int_{0}^{e} \frac{d z}{z \log z \cdots \log _{n-1} z \log _{n}^{1+\epsilon} z}
$$

which converges. Expression (4.2) converges and there is no oscillation. If condition (B) were valid, a similar argument would show that (4.1) converges.

Let $\alpha=\beta$ and assume condition (A). Expression (4.16) is bounded below by zero and (4.17) is greater than

$$
\int_{0}^{\cdot} \frac{d z}{z \log z \cdots \log _{n} z}
$$

which diverges. Hence (4.2) diverges. A similar argument shows that (4.1) diverges. It then follows that the partial sums generated oscillate.

Now let $X$ be a random variable whose distribution function satisfies the assumptions of Theorem 4.2. Let $C(x)$ denote the greatest integer less than or equal to $x$. Consider the two auxiliary lattice random variables

$$
X_{-}=h C(X / h), \quad X_{+}=X_{-}+h,
$$


where $h$ is such that $P\left\{X_{-}=h\right\}, P\left\{X_{+}=h\right\}>0$. Now

$$
X_{-} \leqq X \leqq X_{+} \text {. }
$$

$X_{-}, X, X_{+}$have distribution functions with the same behavior at $-\infty$ and $+\infty$. Therefore $X_{-}, X, X_{+}$have characteristic functions with the same behavior as $z \rightarrow 0$. In view of what has been proved, $X_{-}$and $X_{+}$generate partial sums which either oscillate together or are both positive finitely often with probability one or are both negative finitely often with probability one. Hence, the theorem applies to $X$ in view of (4.18).

Theorem 4.3. Let $F(x)$ be such that

$$
1-F_{2}(x)=x^{-\alpha} h_{2}(x), \quad F_{1}(x)=x^{-\alpha} h_{1}(x), \quad 0<\alpha<1,
$$

where

$$
\lim _{x \rightarrow \infty} \frac{h_{2}(c x)}{h_{2}(x)}=\lim _{x \rightarrow-\infty} \frac{h_{1}(c x)}{h_{1}(x)}=1
$$

for every constant $c>0$. Assume condition (A) and

$$
\lim _{x \rightarrow \infty} \frac{h_{2}(x)}{h_{1}(-x)}=0, \quad\left(\lim _{x \rightarrow \infty} \frac{h_{1}(-x)}{h_{2}(x)}=0\right) .
$$

Then

$$
\begin{aligned}
P\left\{\lim _{k \rightarrow \infty}\left|S_{k}\right|=\infty\right\} & =P\left\{\liminf _{k \rightarrow \infty} S_{k}=-\infty\right\} \\
& =P\left\{\limsup _{k \rightarrow \infty} S_{k}=\infty\right\}=1, \\
\lim _{k \rightarrow \infty} P\left\{S_{k}>0\right\}=0, & \left(\lim _{k \rightarrow \infty} P\left\{S_{k}<0\right\}=0\right)
\end{aligned}
$$

simultaneously.

If condition (A) is valid, the partial sums generated oscillate but have no recurrent values. Whenever there is oscillation and there are no recurrent values (4.19) is true. For then

$$
\sum_{k=1}^{\infty} P\left\{\left|S_{k}\right|<m\right\}<\infty
$$

for all $m>0$ [1] and hence by the theorem of Borel-Cantelli

$$
P\left\{\lim _{k \rightarrow \infty}\left|S_{k}\right|=\infty\right\}=1 \text {. }
$$


The fact that there is oscillation implies that

$$
P\left\{\liminf _{k \rightarrow \infty} S_{k}=-\infty\right\}=P\left\{\limsup _{k \rightarrow \infty} S_{k}=\infty\right\}=1 .
$$

Now assume

$$
\lim _{x \rightarrow \infty} \frac{h_{2}(x)}{h_{1}(-x)}=0
$$

Let $\left\{b_{k}\right\}$ be such that

$$
F_{1}\left(-b_{k}\right) \sim 1 / k
$$

as $k+\infty$. Then

$$
1-F_{2}\left(b_{k}\right)=o(1 / k)
$$

as $k \rightarrow \infty$ and

$$
P\left\{S_{k}<x b_{k}\right\} \rightarrow H_{\alpha}(x)
$$

as $n \rightarrow \infty$ by [2]. But $H_{\alpha}(x)$ is the distribution function of a random variable that is nonpositive. Hence

$$
\lim _{k \rightarrow \infty} P\left\{S_{k}>0\right\}=0 .
$$

A similar argument shows that

$$
\lim _{x \rightarrow \infty} \frac{h_{1}(-x)}{h_{2}(x)}=0
$$

implies that

$$
\begin{gathered}
\lim _{k \rightarrow \infty} P\left\{S_{k}<0\right\}=0 . \\
\text { REFERENCES }
\end{gathered}
$$

\section{REFERENCES}

1. K. L. Chung and W. H. J. Fuchs, On the distribution of sums of random variables, Memoirs of the American Mathematical Society, No. 6, 1951, pp. 1-12.

2. W. Doeblin, Sur l'ensemble de puissances d'une loi de probabilité, Studia Mathematica vol. 9 (1941) pp. 71-96.

3. W. Feller, Fluctuation theory of recurrent events, Trans. Amer. Math. Soc. vol. 67 (1949) pp. 98-119.

4. P. Lévy, Théorie de l'addition de variables aleatoires, Paris, 1937.

University of Chicago,

ChicAgo, Ill. 